Acta Crystallographica Section E

Structure Reports

Online

ISSN 1600-5368

\title{
A second orthorhombic polymorph of 2-(pyridin-4-ylmethoxy)phenol. Corrigendum
}

\section{Guang-Tu Wang, ${ }^{a}$ Yong Zhang, ${ }^{a}$ Jin-Xin Yang, ${ }^{a}$ Ping Zou ${ }^{a}$ * and Guang-Feng $\mathrm{Hou}^{\mathrm{b}}$}

${ }^{a}$ College of Life Science, Sichuan Agriculture University, Ya'an 625014, People's Republic of China, and ${ }^{\mathbf{b}}$ Engineering Research Center of Pesticides of Heilongjiang University, Heilongjiang University, Harbin 150050, People's Republic of China

Correspondence e-mail: hgf1000@163.com

Received 31 May 2012; accepted 15 June 2012

The title and Scheme in the paper by Wang et al. [Acta Cryst. (2012), E68, o1366] are corrected.

In the paper by Wang et al. (2012), the chemical name is incorrect in the title and, as a result, the statement in the title is also incorrect. The correct title should be "4-(Pyridin-4ylmethoxy)phenol'. The correct Scheme is shown below.

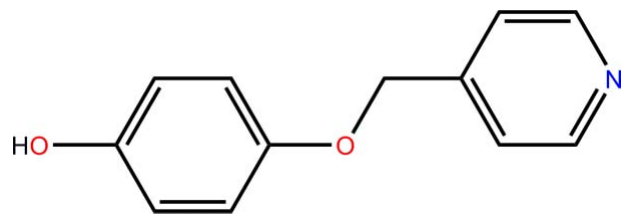

\section{References}

Wang, G.-T., Zhang, Y., Yang, J.-X., Zou, P. \& Hou, G.-F. (2012). Acta Cryst. E68, o1366. 
Acta Crystallographica Section E

\section{Structure Reports}

Online

ISSN 1600-5368

\section{A second orthorhombic polymorph of 2-(pyridin-4-ylmethoxy)phenol}

\section{Guang-Tu Wang, ${ }^{a}$ Yong Zhang, ${ }^{a}$ Jin-Xin Yang, ${ }^{a}$ Ping Zou ${ }^{a}$ * and Guang-Feng $\mathrm{Hou}^{\mathrm{b}}$}

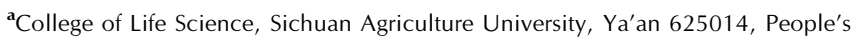
Republic of China, and ${ }^{\mathbf{b}}$ Engineering Research Center of Pesticides of Heilongjiang University, Heilongjiang University, Harbin 150050, People's Republic of China

Correspondence e-mail: hgf1000@163.com

Received 23 March 2012; accepted 31 March 2012

Key indicators: single-crystal X-ray study; $T=293 \mathrm{~K}$; mean $\sigma(\mathrm{C}-\mathrm{C})=0.003 \AA$; $R$ factor $=0.043 ; w R$ factor $=0.077 ;$ data-to-parameter ratio $=16.6$.

The crystal structure of the title compound, $\mathrm{C}_{12} \mathrm{H}_{11} \mathrm{NO}_{2}$, represents a new orthorhombic polymorph II of the previously reported orthorhombic form I [Zhang et al. (2009) Acta Cryst. E65, o3160]. In polymorph II, the six-membered rings form a dihedral angle of $13.8(1)^{\circ}\left[71.6(1)^{\circ}\right.$ in $\left.\mathrm{I}\right]$, and $\mathrm{O}-\mathrm{H} \cdots \mathrm{N}$ hydrogen bonds link molecules into chains along [100], whereas the crystal structure of I features hydrogen-bonded centrosymmetric dimers.

\section{Related literature}

For details of the synthesis, see: Gao et al. (2004). For the crystal structure of polymorph I, see: Zhang et al. (2009).

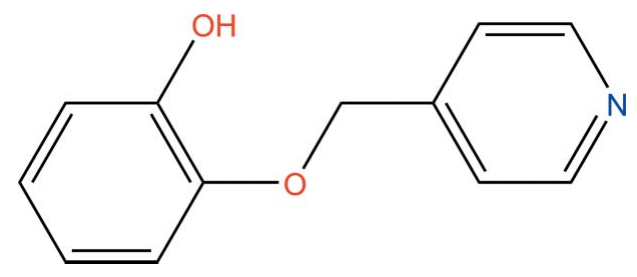

\section{Experimental}

Crystal data
$\mathrm{C}_{12} \mathrm{H}_{11} \mathrm{NO}_{2}$
$M_{r}=201.22$

Orthorhombic, $\mathrm{Pca}_{1}$

$a=23.398(5) \AA$

$b=5.8343$ (12) $\AA$

$c=7.3934(15) \AA$

$V=1009.3(4) \AA^{3}$

$Z=4$

Mo $K \alpha$ radiation

$\mu=0.09 \mathrm{~mm}^{-1}$

$T=293 \mathrm{~K}$

$0.50 \times 0.37 \times 0.11 \mathrm{~mm}$

Data collection

Rigaku R-AXIS RAPID diffractometer

Absorption correction: multi-scan (ABSCOR; Higashi, 1995)

$T_{\min }=0.956, T_{\max }=0.990$

Refinement

$R\left[F^{2}>2 \sigma\left(F^{2}\right)\right]=0.043$

$w R\left(F^{2}\right)=0.077$

$S=1.01$

2285 reflections

138 parameters

8907 measured reflections 2285 independent reflections 1298 reflections with $I>2 \sigma(I)$ $R_{\text {int }}=0.066$

Table 1

Hydrogen-bond geometry $\left(\AA{ }^{\circ}\right)$.

\begin{tabular}{lllll}
\hline$D-\mathrm{H} \cdots A$ & $D-\mathrm{H}$ & $\mathrm{H} \cdots A$ & $D \cdots A$ & $D-\mathrm{H} \cdots A$ \\
\hline $\mathrm{O} 1-\mathrm{H} 1 \cdots \mathrm{N} 1{ }^{\mathrm{i}}$ & 0.82 & 1.95 & $2.763(2)$ & 173 \\
\hline
\end{tabular}

Symmetry code: (i) $x+\frac{1}{2},-y, z$.

Data collection: RAPID-AUTO (Rigaku, 1998); cell refinement: RAPID-AUTO; data reduction: CrystalClear (Rigaku/MSC, 2002); program(s) used to solve structure: SHELXS97 (Sheldrick, 2008); program(s) used to refine structure: SHELXL97 (Sheldrick, 2008); molecular graphics: SHELXTL (Sheldrick, 2008); software used to prepare material for publication: SHELXL97.

This work was supported by a grant from the Two-Way Support Programs of Sichuan Agricultural University (project Nos. 00770115 \& 00770116).

Supplementary data and figures for this paper are available from the IUCr electronic archives (Reference: CV5271).

\section{References}

Gao, C.-M., Cao, D. \& Zhu, L. (2004). Photogr. Sci. Photochem. 22, 103-107. Higashi, T. (1995). ABSCOR. Rigaku Corporation, Tokyo, Japan. Rigaku (1998). RAPID-AUTO. Rigaku Corporation, Tokyo, Japan.

Rigaku/MSC (2002). CrystalClear. Rigaku/MSC Inc., The Woodlands, Texas, USA.

Sheldrick, G. M. (2008). Acta Cryst. A64, 112-122.

Zhang, Z., Li, Y.-J. \& Gao, X.-M. (2009). Acta Cryst. E65, o3160. 


\section{supporting information}

Acta Cryst. (2012). E68, o1366 [doi:10.1107/S1600536812014067]

\section{A second orthorhombic polymorph of 2-(pyridin-4-ylmethoxy)phenol \\ Guang-Tu Wang, Yong Zhang, Jin-Xin Yang, Ping Zou and Guang-Feng Hou}

\section{S1. Comment}

The reported here crystal structure of the title compound, $\mathrm{C}_{12} \mathrm{H}_{11} \mathrm{NO}_{2}$, represents a new orthorhombic polymorph (II)of the previously reported orthorhombic form (I) (Zhang et al. 2009). It was crystalized from a methanol solution of the title compound and (R)-2-(4-(carboxymethoxy)phenoxy)propanoic acid mixture.

In polymorph II (Figure 1), two six-membered rings form a dihedral angle of $13.8(1)^{\circ}\left[71.6(1)^{\circ}\right.$ in I], and intermolecular $\mathrm{O}-\mathrm{H} \cdots \mathrm{N}$ hydrogen bonds link molecules into chains along [100] (Figure 2, Table 1), in spite of hydrogenbonded centrosymmetric dimers in polymorph I.

\section{S2. Experimental}

The 2-(pyridin-4-ylmethoxy)phenol was synthesized by the reaction of $o$-benzenediol and 4-chloromethylpyridine hydrochloride under nitrogen atmosphere and alkaline condition (Gao et al., 2004). Colourless block crystals were obtained by slow evaporation of a methanol solution $(10 \mathrm{~mL})$ containing title compound $(0.402 \mathrm{~g}, 2 \mathrm{mmol})$ and $(R)$-2-(4-(carboxymethoxy)phenoxy)propanoic acid $(0.48 \mathrm{~g}, 2 \mathrm{mmol})$ which only contained the molecules of title compound.

\section{S3. Refinement}

$\mathrm{H}$ atoms bound to $\mathrm{C}$ atoms and the $\mathrm{H}$ atoms of the hydroxyl groups were placed in calculated positions and treated as riding on their parent atoms, with $\mathrm{C}-\mathrm{H}=0.93 \AA$ (aromatic), $\mathrm{C}-\mathrm{H}=0.97 \AA$ (methylene), $\mathrm{O}-\mathrm{H}=0.82 \AA$ and with $\mathrm{U}_{\text {iso }}(\mathrm{H})=1.2 \mathrm{U}_{\mathrm{eq}}(\mathrm{C}), \mathrm{U}_{\mathrm{iso}}(\mathrm{H})=1.5 \mathrm{U}_{\mathrm{eq}}(\mathrm{O})$.

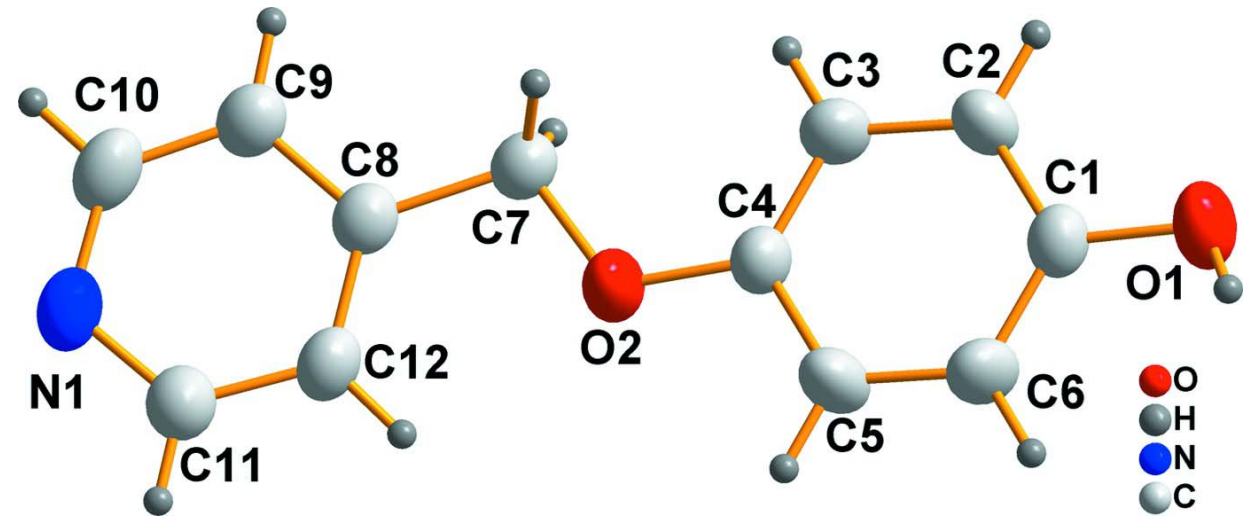

\section{Figure 1}

The molecular structure of the title compound, showing displacement ellipsoids at the $50 \%$ probability level for non- $\mathrm{H}$ atoms. 


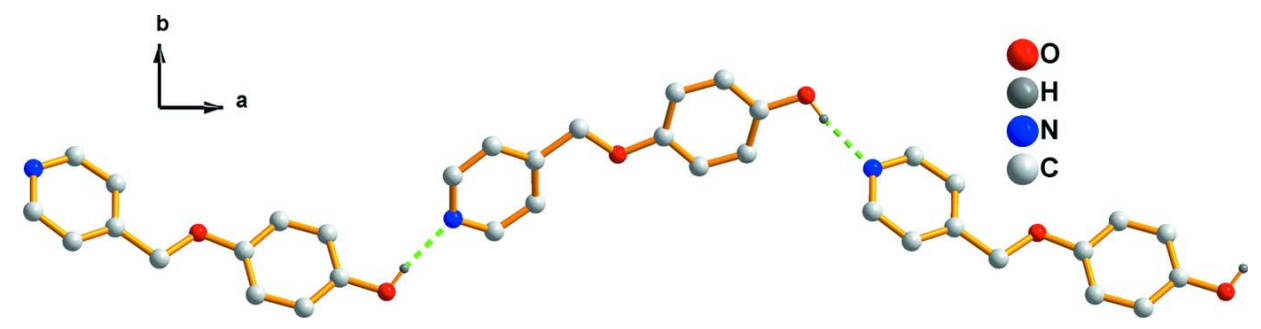

Figure 2

A partial packing view, showing hydrogen-bonded (dashed lines) chain structure along [100].

\section{2-(pyridin-4-ylmethoxy)phenol}

Crystal data

$\mathrm{C}_{12} \mathrm{H}_{11} \mathrm{NO}_{2}$

$M_{r}=201.22$

Orthorhombic, $\mathrm{Pca}_{1}$

Hall symbol: P 2c -2ac

$a=23.398$ (5) $\AA$

$b=5.8343(12) \AA$

$c=7.3934(15) \AA$

$V=1009.3(4) \AA^{3}$

$Z=4$

\section{Data collection}

Rigaku R-AXIS RAPID diffractometer

Radiation source: fine-focus sealed tube

Graphite monochromator

$\omega$ scan

Absorption correction: multi-scan

(ABSCOR; Higashi, 1995)

$T_{\min }=0.956, T_{\max }=0.990$

\section{Refinement}

Refinement on $F^{2}$

Least-squares matrix: full

$R\left[F^{2}>2 \sigma\left(F^{2}\right)\right]=0.043$

$w R\left(F^{2}\right)=0.077$

$S=1.01$

2285 reflections

138 parameters

1 restraint

Primary atom site location: structure-invariant direct methods

Secondary atom site location: difference Fourier

map

Special details

Geometry. All esds (except the esd in the dihedral angle between two 1.s. planes) are estimated using the full covariance matrix. The cell esds are taken into account individually in the estimation of esds in distances, angles and torsion angles; correlations between esds in cell parameters are only used when they are defined by crystal symmetry. An approximate (isotropic) treatment of cell esds is used for estimating esds involving l.s. planes.
$F(000)=424$

$D_{\mathrm{x}}=1.324 \mathrm{Mg} \mathrm{m}^{-3}$

Mo $K \alpha$ radiation, $\lambda=0.71073 \AA$

Cell parameters from 5894 reflections

$\theta=3.3-27.5^{\circ}$

$\mu=0.09 \mathrm{~mm}^{-1}$

$T=293 \mathrm{~K}$

Block, colourless

$0.50 \times 0.37 \times 0.11 \mathrm{~mm}$

8907 measured reflections

2285 independent reflections

1298 reflections with $I>2 \sigma(I)$

$R_{\text {int }}=0.066$

$\theta_{\max }=27.5^{\circ}, \theta_{\min }=3.3^{\circ}$

$h=-30 \rightarrow 30$

$k=-7 \rightarrow 7$

$l=-9 \rightarrow 9$

Hydrogen site location: inferred from neighbouring sites

$\mathrm{H}$-atom parameters constrained

$w=1 /\left[\sigma^{2}\left(F_{\mathrm{o}}^{2}\right)+(0.0218 P)^{2}\right]$

where $P=\left(F_{\mathrm{o}}^{2}+2 F_{\mathrm{c}}^{2}\right) / 3$

$(\Delta / \sigma)_{\max }<0.001$

$\Delta \rho_{\max }=0.15$ e $\AA^{-3}$

$\Delta \rho_{\min }=-0.14$ e $\AA^{-3}$

Extinction correction: SHELXL97 (Sheldrick, 2008), $\mathrm{Fc}^{*}=\mathrm{kFc}\left[1+0.001 \mathrm{xFc}^{2} \lambda^{3} / \sin (2 \theta)\right]^{-1 / 4}$

Extinction coefficient: 0.0028 (6) 
Refinement. Refinement of $\mathrm{F}^{2}$ against ALL reflections. The weighted R-factor $\mathrm{wR}$ and goodness of fit $\mathrm{S}$ are based on $\mathrm{F}^{2}$, conventional R-factors $R$ are based on $F$, with $F$ set to zero for negative $F^{2}$. The threshold expression of $F^{2}>2 \operatorname{sigma}\left(F^{2}\right)$ is used only for calculating R-factors(gt) etc. and is not relevant to the choice of reflections for refinement. R-factors based on $\mathrm{F}^{2}$ are statistically about twice as large as those based on F, and R- factors based on ALL data will be even larger.

Fractional atomic coordinates and isotropic or equivalent isotropic displacement parameters $\left(\AA^{2}\right)$

\begin{tabular}{lllll}
\hline & $x$ & $y$ & $z$ & $U_{\text {iso }} / U_{\text {eq }}$ \\
\hline O1 & $0.68990(6)$ & $0.4700(3)$ & $0.0425(3)$ & $0.0567(5)$ \\
H1 & 0.7109 & 0.3578 & 0.0381 & $0.085^{*}$ \\
O2 & $0.46554(6)$ & $0.1873(3)$ & $-0.0201(2)$ & $0.0566(5)$ \\
N1 & $0.26917(7)$ & $-0.1195(3)$ & $0.0264(3)$ & $0.0488(5)$ \\
C2 & $0.59170(9)$ & $0.5453(4)$ & $0.0842(3)$ & $0.0442(6)$ \\
H2 & 0.6014 & 0.6865 & 0.1339 & $0.053^{*}$ \\
C8 & $0.36646(8)$ & $0.1609(4)$ & $0.0395(3)$ & $0.0390(5)$ \\
C7 & $0.41925(8)$ & $0.3104(4)$ & $0.0562(3)$ & $0.0429(6)$ \\
H7A & 0.4137 & 0.4538 & -0.0078 & $0.052^{*}$ \\
H7B & 0.4269 & 0.3444 & 0.1824 & $0.052^{*}$ \\
C12 & $0.36737(9)$ & $-0.0462(4)$ & $-0.0507(3)$ & $0.0448(6)$ \\
H12 & 0.4003 & -0.0958 & -0.1091 & $0.054^{*}$ \\
C3 & $0.53418(9)$ & $0.4830(4)$ & $0.0713(3)$ & $0.0461(6)$ \\
H3 & 0.5057 & 0.5825 & 0.1107 & $0.055^{*}$ \\
C4 & $0.52013(8)$ & $0.2740(4)$ & $0.0000(3)$ & $0.0410(6)$ \\
C1 & $0.63411(8)$ & $0.4011(4)$ & $0.0247(3)$ & $0.0403(5)$ \\
C6 & $0.61922(9)$ & $0.1936(4)$ & $-0.0516(4)$ & $0.0518(6)$ \\
H6 & 0.6475 & 0.0958 & -0.0948 & $0.062^{*}$ \\
C9 & $0.31568(9)$ & $0.2274(4)$ & $0.1199(3)$ & $0.0463(6)$ \\
H9 & 0.3132 & 0.3668 & 0.1804 & $0.056^{*}$ \\
C10 & $0.26894(9)$ & $0.0849(4)$ & $0.1093(3)$ & $0.0499(7)$ \\
H10 & 0.2351 & 0.1333 & 0.1629 & $0.060^{*}$ \\
C5 & $0.56238(9)$ & $0.1309(4)$ & $-0.0638(4)$ & $0.0541(7)$ \\
H5 & 0.5526 & -0.0089 & -0.1156 & $0.065^{*}$ \\
C11 & $0.31825(8)$ & $-0.1789(4)$ & $-0.0526(3)$ & $0.0485(6)$ \\
H11 & 0.3196 & -0.3186 & -0.1131 & $0.058^{*}$ \\
& & & & \\
\hline
\end{tabular}

Atomic displacement parameters $\left(\AA^{2}\right)$

\begin{tabular}{lllllll}
\hline & $U^{11}$ & $U^{22}$ & $U^{33}$ & $U^{12}$ & $U^{13}$ & $U^{23}$ \\
\hline O1 & $0.0343(8)$ & $0.0535(11)$ & $0.0821(13)$ & $-0.0041(7)$ & $-0.0031(10)$ & $0.0010(11)$ \\
O2 & $0.0315(8)$ & $0.0521(10)$ & $0.0861(14)$ & $-0.0023(7)$ & $0.0054(9)$ & $-0.0184(11)$ \\
N1 & $0.0361(10)$ & $0.0534(13)$ & $0.0569(12)$ & $0.0003(10)$ & $0.0028(10)$ & $0.0084(12)$ \\
C2 & $0.0414(13)$ & $0.0342(13)$ & $0.0569(17)$ & $-0.0032(10)$ & $-0.0020(12)$ & $-0.0076(12)$ \\
C8 & $0.0350(11)$ & $0.0406(13)$ & $0.0416(13)$ & $0.0046(10)$ & $-0.0013(11)$ & $0.0052(12)$ \\
C7 & $0.0378(12)$ & $0.0438(13)$ & $0.0472(15)$ & $0.0026(10)$ & $0.0045(12)$ & $-0.0016(14)$ \\
C12 & $0.0333(12)$ & $0.0500(15)$ & $0.0510(14)$ & $0.0030(11)$ & $0.0049(12)$ & $0.0009(14)$ \\
C3 & $0.0413(13)$ & $0.0392(14)$ & $0.0577(16)$ & $0.0057(11)$ & $0.0009(13)$ & $-0.0070(13)$ \\
C4 & $0.0313(11)$ & $0.0430(13)$ & $0.0487(14)$ & $0.0001(10)$ & $0.0011(10)$ & $-0.0034(12)$ \\
C1 & $0.0329(11)$ & $0.0421(13)$ & $0.0460(14)$ & $-0.0004(10)$ & $0.0000(12)$ & $0.0045(13)$
\end{tabular}


supporting information

\begin{tabular}{lllllll} 
C6 & $0.0375(12)$ & $0.0489(15)$ & $0.0690(17)$ & $0.0089(11)$ & $0.0008(14)$ & $-0.0165(15)$ \\
C9 & $0.0425(13)$ & $0.0437(15)$ & $0.0528(15)$ & $0.0036(12)$ & $0.0052(11)$ & $0.0025(13)$ \\
C10 & $0.0367(13)$ & $0.0554(17)$ & $0.0577(16)$ & $0.0096(12)$ & $0.0054(12)$ & $0.0097(15)$ \\
C5 & $0.0424(13)$ & $0.0429(14)$ & $0.0771(18)$ & $-0.0008(11)$ & $-0.0026(14)$ & $-0.0206(16)$ \\
C11 & $0.0432(12)$ & $0.0516(16)$ & $0.0506(14)$ & $-0.0007(12)$ & $0.0004(12)$ & $-0.0006(14)$ \\
\hline
\end{tabular}

Geometric parameters $\left(\hat{A},{ }^{\circ}\right)$

\begin{tabular}{|c|c|c|c|}
\hline $\mathrm{O} 1-\mathrm{C} 1$ & $1.372(2)$ & $\mathrm{C} 12-\mathrm{C} 11$ & $1.386(3)$ \\
\hline $\mathrm{O} 1-\mathrm{H} 1$ & 0.8200 & $\mathrm{C} 12-\mathrm{H} 12$ & 0.9300 \\
\hline $\mathrm{O} 2-\mathrm{C} 4$ & $1.382(2)$ & $\mathrm{C} 3-\mathrm{C} 4$ & $1.368(3)$ \\
\hline $\mathrm{O} 2-\mathrm{C} 7$ & $1.417(2)$ & $\mathrm{C} 3-\mathrm{H} 3$ & 0.9300 \\
\hline $\mathrm{N} 1-\mathrm{C} 11$ & $1.334(3)$ & $\mathrm{C} 4-\mathrm{C} 5$ & $1.377(3)$ \\
\hline $\mathrm{N} 1-\mathrm{C} 10$ & $1.340(3)$ & $\mathrm{C} 1-\mathrm{C} 6$ & $1.380(3)$ \\
\hline $\mathrm{C} 2-\mathrm{C} 1$ & $1.373(3)$ & $\mathrm{C} 6-\mathrm{C} 5$ & $1.382(3)$ \\
\hline $\mathrm{C} 2-\mathrm{C} 3$ & $1.397(3)$ & C6- $-\mathrm{H} 6$ & 0.9300 \\
\hline $\mathrm{C} 2-\mathrm{H} 2$ & 0.9300 & $\mathrm{C} 9-\mathrm{C} 10$ & $1.376(3)$ \\
\hline $\mathrm{C} 8-\mathrm{C} 12$ & $1.381(3)$ & C9-H9 & 0.9300 \\
\hline $\mathrm{C} 8-\mathrm{C} 9$ & $1.384(3)$ & $\mathrm{C} 10-\mathrm{H} 10$ & 0.9300 \\
\hline $\mathrm{C} 8-\mathrm{C} 7$ & $1.517(3)$ & $\mathrm{C} 5-\mathrm{H} 5$ & 0.9300 \\
\hline C7-H7A & 0.9700 & C11-H11 & 0.9300 \\
\hline C7-H7B & 0.9700 & & \\
\hline $\mathrm{C} 1-\mathrm{O} 1-\mathrm{H} 1$ & 109.5 & $\mathrm{C} 3-\mathrm{C} 4-\mathrm{C} 5$ & $119.98(18)$ \\
\hline $\mathrm{C} 4-\mathrm{O} 2-\mathrm{C} 7$ & $118.54(17)$ & $\mathrm{C} 3-\mathrm{C} 4-\mathrm{O} 2$ & $126.16(19)$ \\
\hline $\mathrm{C} 11-\mathrm{N} 1-\mathrm{C} 10$ & $115.8(2)$ & $\mathrm{C} 5-\mathrm{C} 4-\mathrm{O} 2$ & $113.8(2)$ \\
\hline $\mathrm{C} 1-\mathrm{C} 2-\mathrm{C} 3$ & $121.0(2)$ & $\mathrm{O} 1-\mathrm{C} 1-\mathrm{C} 2$ & $118.5(2)$ \\
\hline $\mathrm{C} 1-\mathrm{C} 2-\mathrm{H} 2$ & 119.5 & $\mathrm{O} 1-\mathrm{C} 1-\mathrm{C} 6$ & $122.45(19)$ \\
\hline $\mathrm{C} 3-\mathrm{C} 2-\mathrm{H} 2$ & 119.5 & $\mathrm{C} 2-\mathrm{C} 1-\mathrm{C} 6$ & 119.08 (19) \\
\hline $\mathrm{C} 12-\mathrm{C} 8-\mathrm{C} 9$ & $117.8(2)$ & $\mathrm{C} 1-\mathrm{C} 6-\mathrm{C} 5$ & $120.1(2)$ \\
\hline $\mathrm{C} 12-\mathrm{C} 8-\mathrm{C} 7$ & $122.01(19)$ & $\mathrm{C} 1-\mathrm{C} 6-\mathrm{H} 6$ & 119.9 \\
\hline $\mathrm{C} 9-\mathrm{C} 8-\mathrm{C} 7$ & $120.2(2)$ & $\mathrm{C} 5-\mathrm{C} 6-\mathrm{H} 6$ & 119.9 \\
\hline $\mathrm{O} 2-\mathrm{C} 7-\mathrm{C} 8$ & $107.36(18)$ & $\mathrm{C} 10-\mathrm{C} 9-\mathrm{C} 8$ & $119.2(2)$ \\
\hline $\mathrm{O} 2-\mathrm{C} 7-\mathrm{H} 7 \mathrm{~A}$ & 110.2 & $\mathrm{C} 10-\mathrm{C} 9-\mathrm{H} 9$ & 120.4 \\
\hline $\mathrm{C} 8-\mathrm{C} 7-\mathrm{H} 7 \mathrm{~A}$ & 110.2 & $\mathrm{C} 8-\mathrm{C} 9-\mathrm{H} 9$ & 120.4 \\
\hline $\mathrm{O} 2-\mathrm{C} 7-\mathrm{H} 7 \mathrm{~B}$ & 110.2 & $\mathrm{~N} 1-\mathrm{C} 10-\mathrm{C} 9$ & $124.1(2)$ \\
\hline $\mathrm{C} 8-\mathrm{C} 7-\mathrm{H} 7 \mathrm{~B}$ & 110.2 & $\mathrm{~N} 1-\mathrm{C} 10-\mathrm{H} 10$ & 118.0 \\
\hline $\mathrm{H} 7 \mathrm{~A}-\mathrm{C} 7-\mathrm{H} 7 \mathrm{~B}$ & 108.5 & $\mathrm{C} 9-\mathrm{C} 10-\mathrm{H} 10$ & 118.0 \\
\hline $\mathrm{C} 8-\mathrm{C} 12-\mathrm{C} 11$ & $118.8(2)$ & $\mathrm{C} 4-\mathrm{C} 5-\mathrm{C} 6$ & $120.5(2)$ \\
\hline $\mathrm{C} 8-\mathrm{C} 12-\mathrm{H} 12$ & 120.6 & $\mathrm{C} 4-\mathrm{C} 5-\mathrm{H} 5$ & 119.7 \\
\hline $\mathrm{C} 11-\mathrm{C} 12-\mathrm{H} 12$ & 120.6 & $\mathrm{C} 6-\mathrm{C} 5-\mathrm{H} 5$ & 119.7 \\
\hline $\mathrm{C} 4-\mathrm{C} 3-\mathrm{C} 2$ & $119.3(2)$ & $\mathrm{N} 1-\mathrm{C} 11-\mathrm{C} 12$ & $124.4(2)$ \\
\hline $\mathrm{C} 4-\mathrm{C} 3-\mathrm{H} 3$ & 120.3 & $\mathrm{~N} 1-\mathrm{C} 11-\mathrm{H} 11$ & 117.8 \\
\hline $\mathrm{C} 2-\mathrm{C} 3-\mathrm{H} 3$ & 120.3 & $\mathrm{C} 12-\mathrm{C} 11-\mathrm{H} 11$ & 117.8 \\
\hline
\end{tabular}




\section{supporting information}

Hydrogen-bond geometry $\left(A,{ }^{\circ}\right)$

\begin{tabular}{lllll}
\hline$D-\mathrm{H} \cdots A$ & $D-\mathrm{H}$ & $\mathrm{H} \cdots A$ & $D \cdots A$ & $D-\mathrm{H} \cdots A$ \\
\hline $\mathrm{O} 1-\mathrm{H} 1 \cdots \mathrm{N} 1^{\mathrm{i}}$ & 0.82 & 1.95 & $2.763(2)$ & 173 \\
\hline
\end{tabular}

Symmetry code: (i) $x+1 / 2,-y, z$. 